\title{
Assumptions of biological measurements: important considerations when evaluating western blot data
}

\author{
Maxwell DeNies ${ }^{1}$, Allen $\mathrm{Liu}^{2}$, and Santiago Schnell ${ }^{1}$ \\ ${ }^{1}$ University of Michigan Medical School \\ ${ }^{2}$ University of Michigan College of Engineering
}

January 23, 2021

\begin{abstract}
As technological and analytical innovations rapidly advance our ability to reveal increasingly complex biological processes, the importance of understanding the assumptions behind biological measurements and sources of uncertainty are essential for data interpretation. This is particularly important in fields such as cell signaling, as due to its importance for both homeostatic and pathogenic biological processes, a quantitative understanding of the basic mechanisms of these transient events is fundamental to drug development. While developed decades ago, western blotting remains an indispensible research tool to probe cell signaling, protein expression, and protein-protein interactions. While improvements in statistical and methodology reporting have improved data quality, understanding the basic experimental assumptions and visual inspection of western blots provides additional information that is useful when evaluating experimental conclusions. Using agonist-induced receptor posttranslational modification as an example we highlight the assumptions of western blotting and showcase how clues from raw western blots can hint at experimental variability that is not captured by statistics and methods that influences quantification. The purpose of this article is not to serve as a detailed review of the technical nuances and caveats of western blotting. Instead using an example we illustrate how experimental assumptions, design, and data normalization can be identified in raw data and influence data interpretation.
\end{abstract}

\section{INTRODUCTION}

The ability to measure and determine the uncertainty of measurements is fundamental to all science. Quantities in cell biology are inherently small, but vary across multiple scales. For instance, intracellular protein concentrations range from $\mathrm{pM}$ to $\mathrm{nM}$, length of cellular structures range from $\mathrm{nm}$ to $\mu \mathrm{m}$, forces generated by molecular motors in the $\mathrm{pN}$ range, and timescale of signaling transduction events from sub-seconds to hours. Biological systems also interact in complicated ways with their internal and external environment, and are dynamically changing making specific attributes difficult to measure. As a consequence, biological measurements often result in a distribution of values. Coupled with biological complexity and the observational nature of the biological sciences, much of our current knowledge of molecular mechanisms is largely semi-quantitative. Recent advancements in -omics approaches as well as technical and analytical improvements in microscopy, flow cytometry, and western blotting have ushered in a new era of cell biology in which increasingly high-throughput screening and data collection are rapidly becoming feasible and minute differences between single cells increasingly quantifiable.

A measurement consists of two parts: a value determined by a particular method as well as the uncertainty of the measured value (Plant et al. 2018; 2014). While uncertainty is commonly defined as the experimental variability in a measurement between replicates, in theory it is additive of all uncertainties throughout the experimental process (BIPM 2008). One often overlooked source of uncertainty as highlighted in the Guide to Expression of Uncertainty in Measurement (GUM), is the incomplete definition of the measurand (thing being measured) and assumptions about what is being measured (BIPM 2008). Additionally, the possibility 
of non-representative or incomplete sampling of what was intended to be measured as well as an incomplete definition of the measurand are common sources of uncertainty defined within the GUM (BIPM 2008). While these additional sources of uncertainty are not always readily apparent in biological systems, the inherent nature of biological measurements makes understanding these sources of uncertainty critically important when interpreting research results.

While new tools are continually developed to detect protein abundance, post-translational modification (PTM) and cell signaling - flow cytometry, FRET biosensors, microscopy, etc. (Miura, Matsuda, and Aoki 2014; Malik et al. 2012; Kelsie Eichel and Zastrow 2018; Davies et al. 2016; Harvey et al. 2008) - due to cost and easy implementation, for almost a half-century, western blotting has been and remains an indispensable tool for bioengineers and biologists (Burnette 2009). In this perspective we take a closer look at several factors that may influence western blot interpretation. The purpose of this perspective is not to review the best practices for western blotting. Several exceptional articles have previously delved into these issues in great detail and readers are strongly encouraged to read if interested (Janes 2015; Eaton et al. 2014). Instead we focus on assumptions and aspects of western blots that experimentalists should consider when evaluating data.

\section{EXPERIMENTAL ASSUMPTIONS OF WESTERN BLOTTING AND HOW THEY CAN INFLUENCE RESULTS}

While statistical analyses and the practice of plotting individual data points improve the accurate portrayal of experimental results, additional information can be gained from looking at raw data. This is particularly true when evaluating western blots or immunofluorescence images. One example from our research that showcases several important factors to consider when evaluating western blots is shown in Figure 1a. The goal of this experiment was to investigate how CXCR4 (C-X-C chemokine receptor 4) mutation (S338/39A and S324/25A) impacts CXCR4 expression and downstream signaling. In addition to probing for total protein and GAPDH (loading controls), CXCR4 was detected using two antibodies: UMB2 and MYC (Figure 1a). UMB2 is a commercial monoclonal antibody that was initially marketed to detect total CXCR4. However, it has recently been shown to be sensitive to CXCR4 PTMs (DeNies et al. 2019; Mimura-Yamamoto et al. 2017, DeNies et al. 2020). MYC is a polyclonal antibody that detects the MYC epitope tag. Experiments were conducted in retinal pigment epithelial cells (RPE) cells overexpressing individual CXCR4 constructs having a C-terminal MYC tag. Wildtype RPE cells have negligible endogenous CXCR4 expression and are unresponsive to CXCL12 (CXCR4 agonist) (Steel, Murray, and Liu 2014; DeNies et al. 2019). In the following subsections we will present specific situations where consideration of loading controls, experimental timescales, antibody stripping, and antibody banding patterns can influence western blot interpretation.

\section{EXAMINE THE LOADING CONTROLS TO SEE IF EXPERIMENTAL CONDITIONS ARE COMPERABLE}

When initially evaluating a western blot, it is first important to examine the loading control. While loading controls such as GAPDH and actin are still common practice, total protein stain should be used whenever possible (Aldridge et al. 2008; Gilda and Gomes 2013; Dittmer and Dittmer 2006; Eaton et al. 2014). However, when not feasible, loading controls should be confirmed to not fluctuate with experimental treatment (Eaton et al. 2014). In this case, while loading controls appear similar within each signaling time course, WT and mutant CXCR4 (S338/39A and S324/25A) samples are different. While not ideal, loading protein abundance variability is relatively common in experiments where multiple cell lines or $+/$ - protein knockdown are compared due to unaccounted factors such as differences in cell growth. However, this should be considered when interpreting results as it could suggest that cells were not grown under the same conditions, were seeded differently or have differential growth rates. Protein concentration quantification prior to western blotting can be used to ensure equal loading. In this case, unless sample protein concentrations are listed, information about changes growth conditions or cell density are indistinguishable by western blot. A second assumption is that subsequent antibody detection is within the linear range. Details of this are explained in depth in other reviews (Janes 2015; Eaton et al. 2014) and unless western blot bands are clearly oversaturated, it is difficult to assess by inspecting individual western blot without additional information. 


\section{CONSIDER EXPERIMENTAL TIMESCALES WHEN EVALUATING QUANTIFICATION}

For experiments with short timescales in particular (i.e. signaling experiments), a third assumption is that total signaling protein detection remains constant between experimental time points. This is illustrated with the UMB2 antibody detection of CXCR4 (Figure 1). The UMB2 antibody was originally thought to detect total CXCR4, however upon agonist-addition, there is a drastic reduction in CXCR4 detection (Figure 1 - first 4 lanes). There are several explanations for this observation. Firstly, it is possible that CXCR4 is degraded. However, since this phenomenon happens within minutes, degradation is highly unlikely and inconsistent with knowledge of CXCR4 trafficking (DeNies et al. 2019). Alternatively, since CXCR4 stimulus induces receptor internalization, it is possible that CXCR4 is more difficult to extract from different intracellular compartments. However, investigation of CXCR4 detection using a second antibody (MYC) revealed that this is not the case as MYC detection is not lost upon agonist-addition (Figure 1 - first 4 lanes) - this has been confirmed in the literature (DeNies et al. 2019). A third explanation is that upon receptor stimulus there is a change in the ability for the UMB2 antibody to detect CXCR4 which is most likely due to CXCR4 PTM as agonist-induced CXCR4 PTM is well established in the region where the UMB2 antibody recognizes CXCR4 (DeNies et al. 2019; Busillo et al. 2010; Luo et al. 2017; Marchese and Benovic 2001). While the UMB2 antibody has become a useful research tool to study CXCR4 (DeNies et al. 2019; MimuraYamamoto et al. 2017; DeNies et al. 2020), understanding why there is a change in antibody detection can provide useful information regarding quantification accuracy. This phenomenon is not unique to CXCR4 as changes in protein localization, PTM, or structure are often essential for proper protein function. However, understanding experimental design caveats can provide critical information about the underlying biological principles investigated.

\section{CONSIDER HOW WESTERN BLOT STRIPPING CAN INFLUENCE DATA QUANTIFI- CATION}

Incomplete stripping is another common western blotting issue that can lead to inaccurate data quantification. This is particularly true when evaluating signaling cascade activity using phospho-specific antibodies. It is common that phospho and total signaling protein antibodies are raised in the same species - i.e. both are anti-rabbit. Therefore, removal of the phospho-specific antibody is required to obtain both total and phospho-specific band quantifications on the same blot. However, failure to properly remove the phosphospecific antibody can lead to inaccurate quantification. One example of this is shown in Figure 2. In this experiment, we monitored CXCL12-induced ERK1/2 phosphorylation in RPE cells overexpressing WT or mutant CXCR4 (Figure 2). The western blot was imaged both pre- and post-antibody stripping. As expected prior to antibody stripping, CXCL12 robustly induces ERK1/2 phosphorylation (Figure 2). However, while less pronounced, phospho-ERK1/2 staining persists after antibody stripping (Figure 2). As a result, subsequent staining using a total ERK1/2 antibody of the same species or staining for a different protein with a similar molecular weight would lead to inaccurate quantification. One way to bypass this limitation is to use a total-ERK1/2 antibody that is raised in a different species thereby removing the necessity for blot stripping and reprobing (Figure 2). While in this example the presence of incomplete stripping is visible by eye, this is not always the case and can still influence data quantification as primary antibody remains. While the fact that stripping buffers may not remove primary antibodies is commonly acknowledged in stripping buffer specifications, in practice this is readily disregarded. Oftentimes stripping and reprobing blots does not change the overall data interpretation and is necessary due to limited resources (imaging channels) and lack of validated antibodies raised in different species.

One example of this is shown in Figure 1 lanes 1-4. As we established earlier, UMB2 detection is negatively correlated with CXCR4 PTM. Therefore in this scenario the MYC antibody is the total signaling protein antibody and UMB2 is PTM sensitive. Close inspection of the staining shows that there is a visible decrease in MYC detection at later time points (i.e. lane 1 vs. lane 4). This is not biologically relevant as we have previously confirmed that CXCR4 is not degraded or differentially extracted at the 20 min stimulus time point (DeNies et al. 2019). While most of the UMB2 staining is removed by stripping, some residual antibody remains. Therefore, earlier time points with higher UMB2 detection have higher background UMB2 detec- 
tion, which is subsequently detected in the MYC quantification. This breaks the third assumption of western blotting that the total signaling protein is constant during the experiment. In this case, when normalizing UMB2 to MYC detection to quantify CXCR4 PTM, this leads to an underestimate for actual CXCR4 PTM but does not impact the data conclusion - that CXCL12-induces CXCR4 PTM. Only inspection of the raw western blots allows this information to become evident to the reader. One way to quantify the extent of incomplete western blot stripping is to re-probe the freshly stripped blot with secondary antibody and re-image. In theory if all antibody was stripped from the blot, the resulting blot should have no staining. However, if antibodies were not completely removed, a similar banding pattern to the pre-stripped blot would be observed and, as describe in the aforementioned UMB2 and MYC staining example, can influence quantification. While in many experiments this should not impact overall interpretation, in the case of comparisons between WT and mutant proteins or +/- genetic knockdown, this may change data interpretation as well as statistical significance if incomplete stripping is not consistent between experimental samples.

A more realistic experimental situation, where mutant and WT CXCR4 expression and agonist-induced receptor PTM are investigated is illustrated in the subsequent western blot lanes (Figure 1; lanes 5-12). CXCR4 S338/39A and CXCR4 S324/25A are phospho-null mutant receptors of biological relevant serine residues that regulate CXCR4 internalization and signaling (Busillo et al. 2010). As described earlier and illustrated in Figure 1, mutation of just 2 residues in this case can have drastic implications in term of CXCR4 detection using the UMB2 antibody. We found that at the 0 min time point S338/39A mutant UMB2 detection was drastically higher compared to WT, while the S324/25A mutant UMB2 detection was negligible (Figure 1). Superficially, this could suggest that S338/39A increases CXCR4 expression while the S324/25A mutant is poorly expressed. However, investigation again with the MYC antibody clarifies that the S324/25A mutant is expressed (Figure 1; lanes 9-12). Again, there are several hypotheses that may explain why this occurs. It is possible that S324/25A completely prevents CXCR4 UMB2 antibody detection due to a change in structure or leads to a different CXCR4 PTM state the decreased UMB2 antibody affinity. In contrast, S338/39A detection with both the UMB2 and MYC antibody was elevated compared to WT CXCR4 suggesting that these mutations may have attenuated degradation and possibly PTM (Figure 1). Further comparisons of how agonist-induced PTM is influenced by CXCR4 mutation further highlights the difficulty of solely relying on these data for interpretation as incomplete stripping differentially impacts the MYC detection of each of these receptors and decoupling of receptor mutation from CXCR4 detectability is not possible in this data alone (Figure 1). These are important questions that should be considered when evaluating western blots and can only be evaluated by raw data inspection. In this case the interpretation of the presented results are confounding and additional lines of evidences are necessary for interpretation.

\section{EVALUATE ANTIBODY BANDING PATTERNS TO IDENTIFY POTENIAL QUANTIFI- CATION LIMITATIONS}

Another important aspect to consider when evaluating western blots is antibody-banding pattern. While it is well established that protein PTMs such as ubiquitination or phosphorylation can influence protein migration on SDS-PAGE gels, the phospho-specific antibody should detect a subset of the total antibody detection bands - in this example total antibody refers to the total detection of a single protein such as the total ERK1/2 antibody. In our experiment, this condition was met as the UMB2 detection bands are contained within a subset of the MYC (Figure 1). Failure of this assumption can have similar effects to incomplete stripping and inaccurate quantification. Additionally, when evaluating short timescale time course data it is important to consider the experimental approach used to collect data as this can be a source of uncertainty particularly at early time points. Assume that it takes approximately 30 seconds to take cells from the incubator, remove media, and prep samples for protein extraction. In this case, a 1 min stimulus is in practice closer to $1.5 \mathrm{~min}$ - a $50 \%$ increase in stimulus time. At later time points, this is less of an issue as 20.5 min vs. $20 \mathrm{~min}$ is only a $2.5 \%$ increase in stimulus time. Complications arise when collecting data from multiple samples as the order of experiments can lead to unintended but relevant differences in collection time. Therefore, caution should be used when evaluating experiments with short timescales. Among other approaches to mitigate these limitations, conducting all post-experiment processes on ice or using technology (such as microfluidics) to precisely add ligands and lyse cells can be used to improve accuracy (Chylek et al. 
2014).

While the caveats described in this section are illustrated through comparison of WT and mutant CXCR4 PTM, the core assumptions are broadly applicable to other western blotting experiments as well as other techniques such as microscopy.

\section{HOW DO WESTERN BLOTTING ASSUMPTIONS INFLUENCE QUANTIFICATION?}

In the aforementioned section, we highlighted some of the caveats to consider in western blotting experiments. In this section, we will illustrate how they can influence quantification and interpretation. The first step in quantification is data normalization. While technical and experimental variability between experimental replicates make this step necessary, normalization protocols can drastically influence result interpretation. To illustrate this, a simple hypothetical case is illustrated in Figure 3A. In this experiment, total protein loading is assumed constant and we are making a comparison between a control and treatment (i.e. inhibitors, knockdown, mutant protein etc.) group at 0 and 5 min post-stimulus. One approach seen in the literature is to normalize values to the initial pre-treatment sample (Figure 3B (i)). An issue with this approach is that typically pre-treatment (no stimulus in the case) samples have significantly lower signal-to noise-ratios compared to later time points in this case when the intensity is much higher. As illustrated in Figure 3B (i) by doing so, minor changes in the base of the normalization can have significant implications on the relative change in the signal. While the reciprocal approach mitigates many of the concerns of amplifying value uncertainty to other samples as maximum values have a higher signal to noise ratio, this approach does not permit comparisons between maximum values between multiple treatments (Figure 3B (ii)). A third strategy builds on this approach and normalizes samples to the "maximum value time point" of the control experiment. Using this approach, the issue of inter-western blot variability is limited and by normalizing to the maximum or close to maximum value, concerns of amplifying value uncertainty to other time points is greatly reduced (Figure 3B (iii)). Additionally, by normalizing to the maximum value of the control, comparisons between maximum signaling values of different treatments is possible. However, due to the normalization, the uncertainty of the control maximum value is not considered and the assumption that the treatment does not significantly influence other secondary factors such as signaling protein expression need to be considered. While the practice of multiple normalization should be limited whenever possible, when used it is imperative to describe the approach explicitly in figure legends and methods.

\section{OTHER CONSIDERATIONS FOR CELL SIGNALING EXPERIMENTS}

In part due to the recent discoveries of the importance of compartmentalization of receptor signaling, a common approach to study the role of a particular organelle or protein in regulating signaling is to transiently knockdown or knockout a protein of interest and measure the effect of receptor signaling (Garay et al. 2015; English, Mahn, and Marchese 2018; Rosselli-Murai et al. 2018; DeNies et al. 2019; K. Eichel, Jullié, and von Zastrow 2016). However, due to the importance of many of these proteins, potential compensatory pathways, and multiple uses of many of proteins involved in endocytic trafficking it is important to consider secondary affects of knockdowns/knockouts on receptor signaling and attempt to decouple secondary effects when possible (Liberali and Pelkmans 2012). Recent work has estimated that over $50 \%$ of human proteins have multiple intracellular localizations (Thul et al. 2017). One classical example from endocytic trafficking is that clathrin-mediated endocytosis occurs at both the plasma membrane and Golgi apparatus (Wu et al. 2003) (Figure 4). Additionally, recent work from our lab has shown that a ubiquitination-deficient CXCR4 mutant receptor artificially localizes to intracellular compartments (DeNies et al. 2020). Compared to WT, this mutant receptor has reduced signaling and agonist-dependent PTM. However, without additional experiments it would not be possible to determine whether these observations were due to the change in receptor localization or mutation. To attempt to decouple these affects, we generated additional constructs that restored mutant receptor plasma membrane localization to confirm that these defects were indeed due to receptor mutation and not localization (DeNies et al. 2020). Likewise, due to the fundamental importance of signal transduction and receptor regulation, cells have evolved multiple compensatory pathways to control receptor endocytosis. Multiple receptors have been shown to be internalized by both clathrin- and clathrin-independent endocytic mechanisms (Figure 4) (Di Guglielmo et al. 2003; Sigismund et al. 2005) and 
research on Epidermal Growth Factor Receptor (EGFR) has revealed that these changes are to due ligand concentration-dependent receptor PTMs (Sigismund et al. 2005; 2013). Additionally when relating signaling potential back to receptor expression it is also important to consider the spare receptor hypothesis as it has been shown that activation of only a subset of receptors is necessary for maximal signaling (Brown et al. 1992). This is particularly true when evaluating the signaling potential of receptors when treatment (i.e. WT vs. mutant receptor, protein knockdown etc.) affects receptor trafficking or expression. Lastly, it is important to note that truly rigorous and robust science is ideally supported by multiple methodologies and whenever possible, alternative approaches implemented to test initial conclusions (Munafò and Smith 2018; Kaelin Jr 2017).

\section{CONCLUSION}

Innovation, creativity, and pushing the limits of technology and research methodologies are fundamental to scientific innovation. Unfortunately, a byproduct of this process is the increased likelihood of honest yet irreplicable results. The failure to replicate could result from heterogenous factors that include lack of standardization to report and share experimental protocols, poor experimental design statistical problem or biases in hypothesis testing. In this work, we illustrate another important factor that could be playing an important role in the failure to reproduce: the fundamental assumptions made to carry out biological measurements. We believe implementation of core metrology principles within receptor signaling has the potential not only reduce replicability issues but more importantly facilitate discoveries that approach absolute scientific truth. Throughout this article, we have revisited the fundamental assumptions of western blotting and highlighted important features to consider when evaluating western blot data that contribute to data uncertainty.

As the bioengineering and biological sciences become more quantitative, the replicability of measurements between different research groups is the minimum gold standard of science. The culture of replicability requires members of the scientific community to become responsible for the gold standards of reporting measurement protocols and assumptions. These gold standards do not inhibit novelty and significance, but carefully ensure the validity of research findings and whether those finding support the conclusions presented in the research. While western blotting was highlighted in this work, many of the core concepts are applicable to other methodologies used to measure bioengineering, cell signaling and cell biology processes in general.

\section{ACKNOWLEDGEMENTS}

MSD thanks support from the National Science Foundation (Fellow ID: 2015196825). We would like to thank Dr. Justin Eilertsen (University of Michigan), Dr. Wylie Stroberg (University of Alberta) and Hesso Farhan (Medical University of Innsbruck) for constructive discussions. We would also like to thank Mariana Schnell for helping make schematic figures for this publication.

\section{CONFLICT OF INTEREST}

The authors declare that they have no known competing financial interests or personal relationships that could have appeared to influence the work reported in this paper.

\section{DATA AVAILABILITY STATEMENT}

Data sharing is not applicable to this article as no new data were created or analyzed in this study.

\section{Figures}




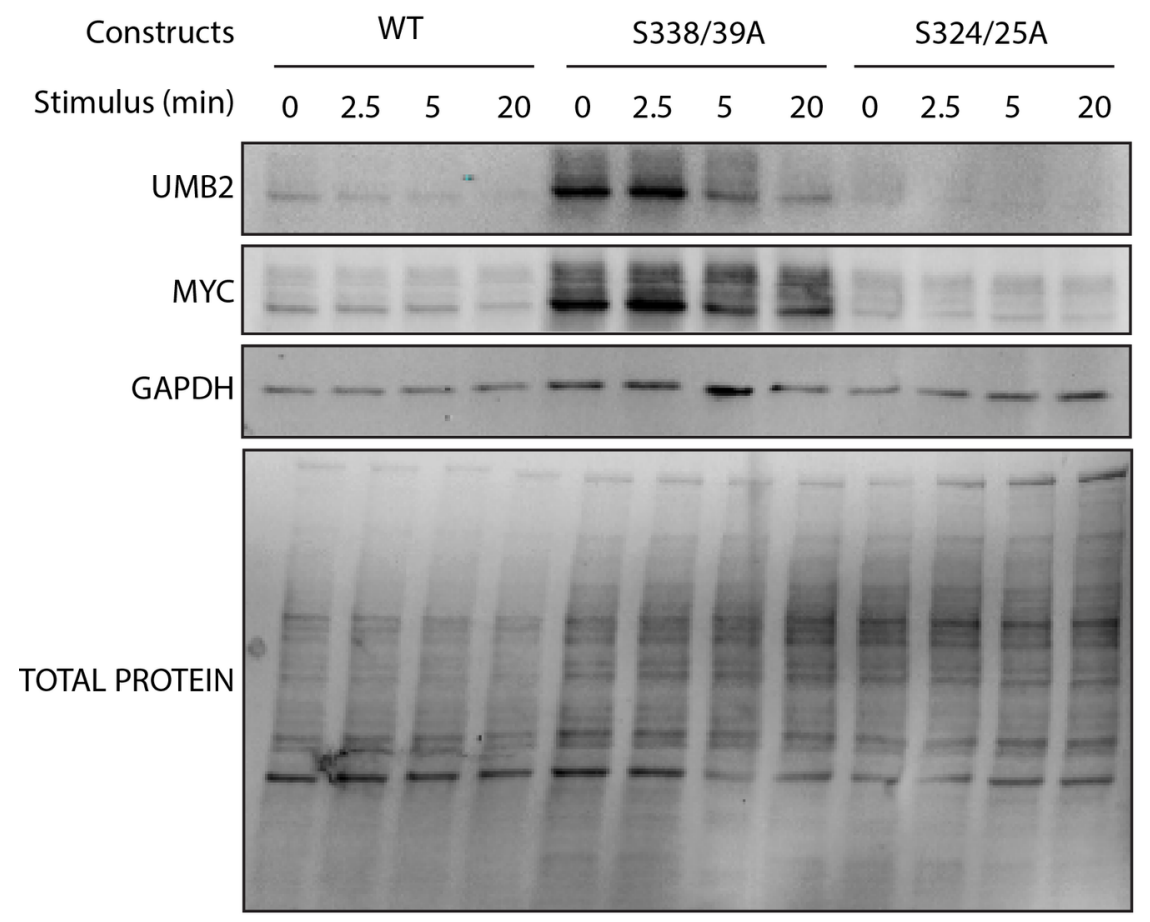

Figure 1: Western blotting assumptions are not always true. Representative western blot of non-posttranslationally modified (UMB2) and total CXCR4 (MYC) detection for WT and serine mutant receptors (S338/39A and S324/25A). Total Protein and GAPDH staining illustrate protein loading for each replicate. Experimental methods: RPE cells overexpressing CXCR4 were grown to $75 \%$ confluence a treated with fresh FBS (10\%) supplemented media (Gibco PN: 11330-032) 24 hours before the experiment. Cells were serum starved for 4 hours and treated with $12.5 \mathrm{nM}$ CXCL12 (R\&D Systems PN: 350-NS-050) for the described time course. Lysates were extracted using RIPA buffer (Pierce PN: 89900) supplemented with protease and phosphatase inhibitors (Thermo Scientific PN: and respectively) and incubated ice for 20 minutes and centrifuged at 16,100g for 45 minutes at $4^{\circ} \mathrm{C}$. Loading buffer supplemented with beta mercaptoethanol was added to denature lysates and samples were loaded on a 4-20\% BioRad gel and transferred onto PVDF membranes using the iBlot system mixed range transfer (Thermo Scientific). Total protein was quantified using the REVERT Total Protein Stain (LiCor PN: 926-11016). Afterwards, blots were blocked with 5\% BSA (Thermo Scientific PN: 37520) in TBST for 1 hour and incubated with primary antibodies ms-GAPDH (1:1000), rb-UMB2 (1:2000) overnight at $4^{\circ} \mathrm{C}$. Blots were incubated with secondary antibodies (Gt anti ms-700, gt anti rb-800) for 1 hour in 5\% BSA in TBST and imaged using the LiCor Odyssey SA Imaging System. Afterwards, blots were stripped using NewBlot stripping buffer (LiCor PN:928-40032) per manufacture instructions and reprobed with rb-MYC antibody (1:5000) as described above. UMB2 antibody 
was purchased from ABCAM (PN: Ab124824), MYC from Bethyl (PN: A190-105A) and GAPDH from Santa Cruz Biotechnology (PN: sc-47724). Secondary antibodies were purchased from Invitrogen (PN: SA535571 and 35518).

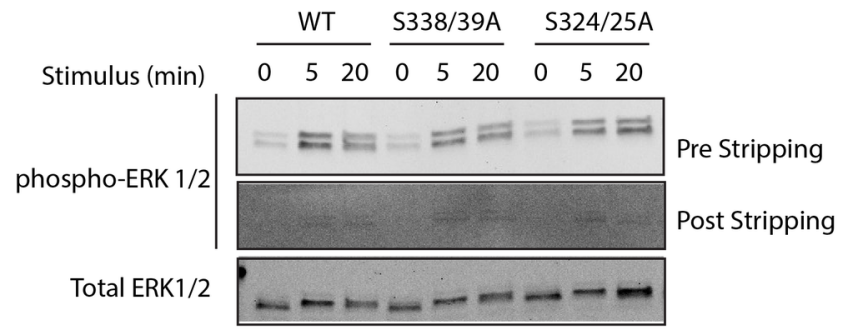

Figure 2: Example of incomplete western blot stripping. Phospho-ERK1/2 detection pre and post-antibody stripping. Total ERK1/2 staining is shown as a loading control. Experimental methods: RPE cells overexpressing WT or mutant CXCR4 were grown to $75 \%$ confluence a treated with fresh FBS (10\%) supplemented media (Gibco PN: 11330-032) 24 hours before the experiment. Cells were serum starved for 4 hours and treated with $12.5 \mathrm{nM}$ CXCL12 (R\&D Systems PN: 350-NS-050) for the described time course. Lysates were extracted using RIPA buffer (Pierce PN: 89900) supplemented with protease and phosphatase inhibitors (Thermo Scientific PN: and respectively) and incubated ice for 20 minutes and centrifuged at 16,100g for 45 minutes at $4^{\circ} \mathrm{C}$. Loading buffer supplemented with beta mercaptoethanol was added to denature lysates and samples were loaded on a 4-20\% BioRad gel and transferred onto PVDF membranes using the iBlot system mixed range transfer (Thermo Scientific). Blots were blocked with 5\% BSA (Thermo Scientific PN: 37520) in TBST for 1 hour and incubated with primary antibodies ms-Total-ERK1/2 (1:1000), rb-phospho-ERK1/2 $(1: 2000)$ in $5 \% \mathrm{BSA}$ in TBST overnight at $4^{\circ} \mathrm{C}$. Blots were incubated with secondary antibodies (Gt anti ms-700, gt anti rb-800) for 1 hour in 5\% BSA in TBST and imaged using the LiCor Odyssey SA Imaging System. Afterwards, blots were stripped using NewBlot stripping buffer (LiCor PN:928-40032) and imaged again to determine antibody stripping efficacy. Total and phospho-ERK1/2 antibodies were purchased from Cell Signaling Technologies (PN: 9107S and 4370S respectively). Secondary antibodies were purchased from Invitrogen (PN: SA535571 and 35518). 
A.

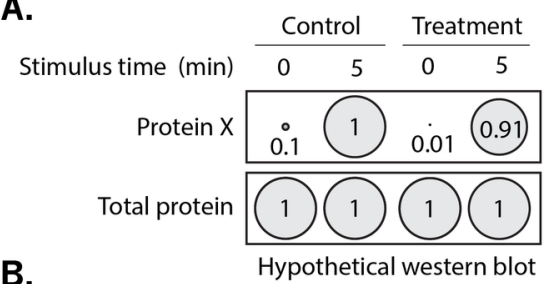

B.

(ii) 5 min norm.

(iii) 5 min control norm.
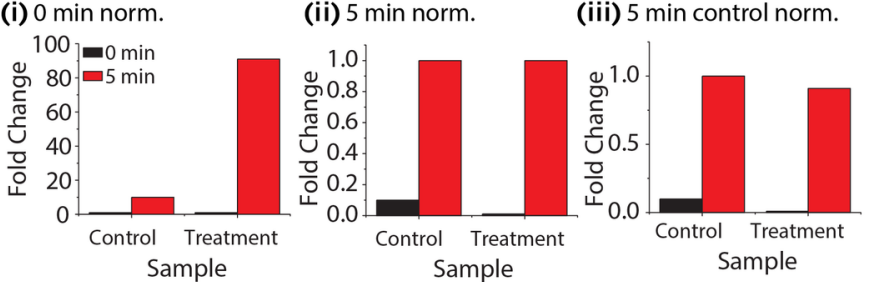

Figure 3: Normalization methodology can influence result interpretation. (A ) Hypothetical western blot results for a control and treatment experiment. Circles diameters are representative of western blot band intensities and are listed. (B ) Quantification of hypothetical western blot results illustrating that normalizing to samples with low signal to noise ratio can propagate error throughout normalization and influence result interpretation. For this representation, noise was assumed to be constant for each sample. (i) Hypothetical quantification of data when normalized to the 0 min time point of each treatment (i.e. normalizing value: 0.1 and 0.01 for the control and treatment respectively). (ii) Hypothetical quantification of data when normalized to 5 minute time point of each condition (i.e. normalizing value: 1 and 0.91 for the control and treatment respectively). (iii) Hypothetical quantification of data when normalized to the 5 minute time point of the control condition (i.e. normalizing value: one for all samples). 


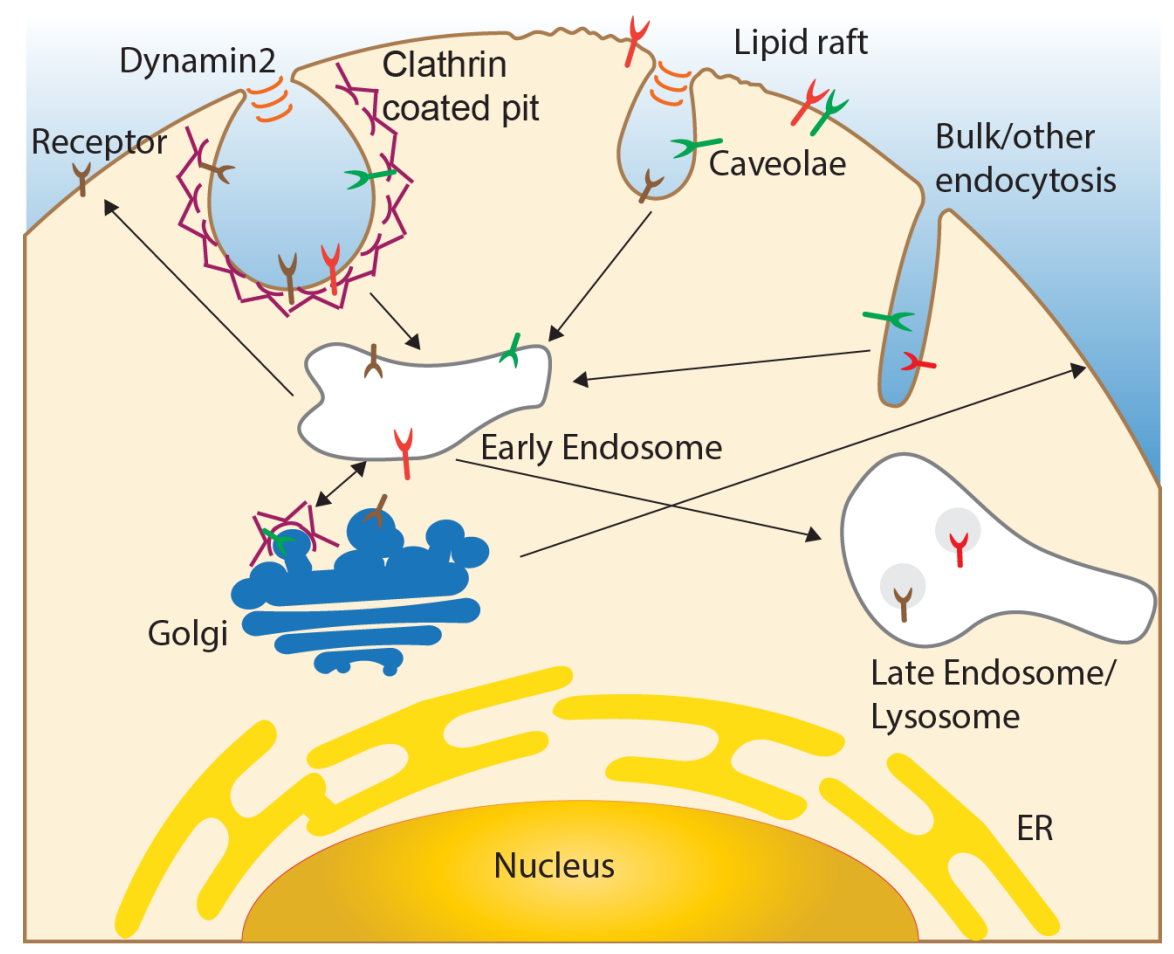

Figure 4: Unintended secondary effects of a treatment can influence result interpretation. A schematic representation of endocytic trafficking that highlights some facts to consider when interpreting receptorsignaling experiments. As illustrated by the schematic, receptors can internalize by different and potentially compensatory endocytic mechanisms. Additionally, clathrin-mediated endocytosis occurs at both the plasma membrane and Golgi, therefore it is important to consider the multiple roles of proteins in cells when conducting isolated knockdown/knockouts experiments as it is possible that the observed effect on cell signaling might be due to a secondary effect of the treatment or genetic perturbation. While this schematic is by no means all encompassing, it highlights some areas that should be considered when designing and interpreting receptor-signaling experiments. Different types of receptors are illustrated in different colors, clathrin triskelia by magenta lattice like structure and dynamin2 by the rope like structure. Internal cellular structures, as well as, the flow of receptors within the cell labeled.

\section{REFERENCES}

Aldridge, Georgina M., David M. Podrebarac, William T. Greenough, and Ivan Jeanne Weiler. 2008. "The Use of Total Protein Stains as Loading Controls: An Alternative to High-Abundance Single-Protein Controls in Semi-Quantitative Immunoblotting." Journal of Neuroscience Methods 172 (2): 250-54. https://doi.org/10.1016/j.jneumeth.2008.05.003.

BIPM. 2008. "BIPM - Guide to the Expression of Uncertainty in Measurement (GUM)." 2008. https://www.bipm.org/en/publications/guides/gum.html.

Brown, L., N. M. Deighton, S. Bals, W. Söhlmann, H. R. Zerkowski, M. C. Michel, and O. E. Brodde. 1992. "Spare Receptors for Beta-Adrenoceptor-Mediated Positive Inotropic Effects of Catecholamines in the Human Heart." Journal of Cardiovascular Pharmacology 19 (2): 222-32. https://doi.org/10.1097/00005344$199202000-00011$.

Burnette, W. Neal. 2009. "Western Blotting : Remembrance of Past Things." Methods in Molecular Biology (Clifton, N.J.) 536: 5-8. https://doi.org/10.1007/978-1-59745-542-8_2. 
Busillo, John M., Sylvain Armando, Rajarshi Sengupta, Olimpia Meucci, Michel Bouvier, and Jeffrey L. Benovic. 2010. "Site-Specific Phosphorylation of CXCR4 Is Dynamically Regulated by Multiple Kinases and Results in Differential Modulation of CXCR4 Signaling." The Journal of Biological Chemistry 285 (10): 7805-17. https://doi.org/10.1074/jbc.M109.091173.

Chylek, Lily A., Vyacheslav Akimov, Jorn Dengjel, Kristoffer T. G. Rigbolt, Bin Hu, William S. Hlavacek, and Blagoy Blagoev. 2014. "Phosphorylation Site Dynamics of Early T-Cell Receptor Signaling." PLoS ONE 9 (8): e104240. https://doi.org/10.1371/journal.pone.0104240.

Davies, Richard, Petra Vogelsang, Roland Jonsson, and Silke Appel. 2016. "An Optimized Multiplex Flow Cytometry Protocol for the Analysis of Intracellular Signaling in Peripheral Blood Mononuclear Cells." Journal of Immunological Methods 436 (September): 58-63. https://doi.org/10.1016/j.jim.2016.06.007.

DeNies, Maxwell S., Luciana K. Rosselli-Murai, Santiago Schnell, and Allen P. Liu. 2019. "Clathrin Heavy Chain Knockdown Impacts CXCR4 Signaling and Post-Translational Modification." Frontiers in Cell and Developmental Biology 7: 77. https://doi.org/10.3389/fcell.2019.00077.

DeNies, Maxwell S., Alan V. Smrcka, Santiago Schnell, and Allen P. Liu. 2020. "ß-Arrestin Mediates Communication between Plasma Membrane and Intracellular GPCRs to Regulate Signaling." Communications Biology 3 (1): 1-12. https://doi.org/10.1038/s42003-020-01510-2.

Di Guglielmo, Gianni M., Christine Le Roy, Anne F. Goodfellow, and Jeffrey L. Wrana. 2003. "Distinct Endocytic Pathways Regulate TGF- $\beta$ Receptor Signalling and Turnover." Nature Cell Biology 5 (5): 410-21. https://doi.org/10.1038/ncb975.

Dittmer, Angela, and Jürgen Dittmer. 2006. " $\beta$-Actin Is Not a Reliable Loading Control in Western Blot Analysis." ELECTROPHORESIS 27 (14): 2844-45. https://doi.org/10.1002/elps.200500785.

Eaton, Samantha L., Maica Llavero Hurtado, Karla J. Oldknow, Laura C. Graham, Thomas W. Marchant, Thomas H. Gillingwater, Colin Farquharson, and Thomas M. Wishart. 2014. "A Guide to Modern Quantitative Fluorescent Western Blotting with Troubleshooting Strategies." Journal of Visualized Experiments : JoVE, no. 93 (November). https://doi.org/10.3791/52099.

Eichel, K., D. Jullié, and M. von Zastrow. 2016. " $\beta$-Arrestin Drives MAP Kinase Signalling from Clathrin-Coated Structures after GPCR Dissociation." Nature Cell Biology 18 (3): 303-10. https://doi.org/10.1038/ncb3307.

Eichel, Kelsie, and Mark von Zastrow. 2018. "Subcellular Organization of GPCR Signaling." Trends in Pharmacological Sciences 39 (2): 200-208. https://doi.org/10.1016/j.tips.2017.11.009.

English, Elizabeth J., Sarah A. Mahn, and Adriano Marchese. 2018. "Endocytosis Is Required for C-X-C Chemokine Receptor Type 4 (CXCR4)-Mediated Akt Activation and Anti-Apoptotic Signaling." Journal of Biological Chemistry, June, jbc.RA118.001872. https://doi.org/10.1074/jbc.RA118.001872.

Garay, Camilo, Gurjeet Judge, Stefanie Lucarelli, Stephen Bautista, Rohan Pandey, Tanveer Singh, and Costin N. Antonescu. 2015. "Epidermal Growth Factor-Stimulated Akt Phosphorylation Requires Clathrin or ErbB2 but Not Receptor Endocytosis." Molecular Biology of the Cell 26 (19): 3504-19. https://doi.org/10.1091/mbc.E14-09-1412.

Gilda, Jennifer E., and Aldrin V. Gomes. 2013. "Stain-Free Total Protein Staining Is a Superior Loading Control to $\beta$-Actin for Western Blots." Analytical Biochemistry 440 (2): 186-88. https://doi.org/10.1016/j.ab.2013.05.027.

Harvey, Christopher D., Anka G. Ehrhardt, Cristina Cellurale, Haining Zhong, Ryohei Yasuda, Roger J. Davis, and Karel Svoboda. 2008. "A Genetically Encoded Fluorescent Sensor of ERK Activity." Proceedings of the National Academy of Sciences 105 (49): 19264-69. https://doi.org/10.1073/pnas.0804598105. 
Janes, Kevin A. 2015. "An Analysis of Critical Factors for Quantitative Immunoblotting." Sci. Signal. 8 (371): rs2-rs2. https://doi.org/10.1126/scisignal.2005966.

Kaelin Jr, William G. 2017. "Publish Houses of Brick, Not Mansions of Straw." Nature News 545 (7655): 387. https://doi.org/10.1038/545387a.

Liberali, Prisca, and Lucas Pelkmans. 2012. "Towards Quantitative Cell Biology." Comments and Opinion. Nature Cell Biology. December 1, 2012. https://doi.org/10.1038/ncb2648.

Luo, Jiansong, John M. Busillo, Ralf Stumm, and Jeffrey L. Benovic. 2017. "G Protein-Coupled Receptor Kinase 3 and Protein Kinase C Phosphorylate the Distal C-Terminal Tail of the Chemokine Receptor CXCR4 and Mediate Recruitment of $\beta$-Arrestin." Molecular Pharmacology91 (6): 554-66. https://doi.org/10.1124/mol.116.106468.

Malik, Rohit, Unice J. K. Soh, JoAnn Trejo, and Adriano Marchese. 2012. "Novel Roles for the E3 Ubiquitin Ligase Atrophin-Interacting Protein 4 and Signal Transduction Adaptor Molecule 1 in G Protein-Coupled Receptor Signaling." The Journal of Biological Chemistry 287 (12): 9013-27. https://doi.org/10.1074/jbc.M111.336792.

Marchese, Adriano, and Jeffrey L. Benovic. 2001. "Agonist-Promoted Ubiquitination of the G ProteinCoupled Receptor CXCR4 Mediates Lysosomal Sorting." Journal of Biological Chemistry 276 (49): 4550912. https://doi.org/10.1074/jbc.C100527200.

Mimura-Yamamoto, Yuka, Hiroshi Shinohara, Taichi Kashiwagi, Toru Sato, Seiji Shioda, and Tatsunori Seki. 2017. "Dynamics and Function of CXCR4 in Formation of the Granule Cell Layer during Hippocampal Development." Scientific Reports 7 (1): 5647. https://doi.org/10.1038/s41598-017-05738-7.

Miura, Haruko, Michiyuki Matsuda, and Kazuhiro Aoki. 2014. "Development of a FRET Biosensor with High Specificity for Akt." Cell Structure and Function 39 (1): 9-20.

Munafò, Marcus R., and George Davey Smith. 2018. "Robust Research Needs Many Lines of Evidence." Nature 553 (7689): 399. https://doi.org/10.1038/d41586-018-01023-3.

Plant, Anne L., Chandler A. Becker, Robert J. Hanisch, Ronald F. Boisvert, Antonio M. Possolo, and John T. Elliott. 2018. "How Measurement Science Can Improve Confidence in Research Results." PLoS Biology 16 (4): e2004299. https://doi.org/10.1371/journal.pbio.2004299.

Plant, Anne L., Laurie E. Locascio, Willie E. May, and Patrick D. Gallagher. 2014. "Improved Reproducibility by Assuring Confidence in Measurements in Biomedical Research." Nature Methods 11 (9): 895-98. https://doi.org/10.1038/nmeth.3076.

Rosselli-Murai, Luciana K., Joel A. Yates, Sei Yoshida, Julia Bourg, Kenneth K. Y. Ho, Megan White, Julia Prisby, et al. 2018. "Loss of PTEN Promotes Formation of Signaling-Capable Clathrin-Coated Pits." Journal of Cell Science 131 (8). https://doi.org/10.1242/jcs.208926.

Sigismund, Sara, Veronica Algisi, Gilda Nappo, Alexia Conte, Roberta Pascolutti, Alessandro Cuomo, Tiziana Bonaldi, et al. 2013. "Threshold-Controlled Ubiquitination of the EGFR Directs Receptor Fate." The EMBO Journal 32 (15): 2140-57. https://doi.org/10.1038/emboj.2013.149.

Sigismund, Sara, Tanja Woelk, Claudia Puri, Elena Maspero, Carlo Tacchetti, Pietro Transidico, Pier Paolo Di Fiore, and Simona Polo. 2005. "Clathrin-Independent Endocytosis of Ubiquitinated Cargos." Proceedings of the National Academy of Sciences of the United States of America 102 (8): 2760-65. https://doi.org/10.1073/pnas.0409817102.

Steel, Elisabeth, Victoria L. Murray, and Allen P. Liu. 2014. "Multiplex Detection of Homo- and Heterodimerization of g Protein-Coupled Receptors by Proximity Biotinylation." PloS One9 (4): e93646. https://doi.org/10.1371/journal.pone.0093646. 
Thul, Peter J., Lovisa Åkesson, Mikaela Wiking, Diana Mahdessian, Aikaterini Geladaki, Hammou Ait Blal, Tove Alm, et al. 2017. "A Subcellular Map of the Human Proteome." Science (New York, N.Y.)356 (6340). https://doi.org/10.1126/science.aal3321.

Wu, Xufeng, Xiaohong Zhao, Rosa Puertollano, Juan S. Bonifacino, Evan Eisenberg, and Lois E. Greene. 2003. "Adaptor and Clathrin Exchange at the Plasma Membrane and Trans-Golgi Network." Molecular Biology of the Cell 14 (2): 516-28. https://doi.org/10.1091/mbc.E02-06-0353. 